\title{
Criminologie
}

\section{Jeunes contrevenants à la croisée des chemins}

\section{Étude à devis mixte du risque suicidaire}

\section{Young offenders at a crossroads}

\section{A study of their suicide risk}

\section{Jóvenes delincuentes en la encrucijada Estudio de estimación mixta del riesgo suicida}

\section{Catherine Laurier, Anne-Marie Ducharme, Laurie St-Pierre et Janet Sarmiento}

Volume 51, numéro 2, automne 2018

Prise en charge du suicide : entre crime, troubles mentaux et droit de mourir

URI : https://id.erudit.org/iderudit/1054244ar

DOI : https://doi.org/10.7202/1054244ar

Aller au sommaire du numéro

Éditeur(s)

Les Presses de l’Université de Montréal

ISSN

0316-0041 (imprimé)

1492-1367 (numérique)

Découvrir la revue

Citer cet article

Laurier, C., Ducharme, A.-M., St-Pierre, L. \& Sarmiento, J. (2018). Jeunes contrevenants à la croisée des chemins : étude à devis mixte du risque suicidaire. Criminologie, 51(2), 288-313. https://doi.org/10.7202/1054244ar
Résumé de l'article

En raison de la prédominance des conduites antisociales des jeunes contrevenants en général et de ceux associés aux gangs de rue en particulier, qui constituent d'ailleurs le motif de leur prise en charge par le système de justice, il est moins fréquent de se pencher sur leur victimisation et leurs séquelles psychologiques. Ainsi, dans le cadre de cet article, 212 jeunes contrevenants, pris en charge par les services jeunesse ou les services correctionnels ont été évalués à l'aide de questionnaires quantitatifs et lors d'une entrevue qualitative semi-structurée. Cette étude à devis mixte a permis de relever, dans la partie quantitative, les variables qui contribuent statistiquement le plus au risque suicidaire, tel qu'il a été évalué à l'aide de deux outils (MAYSI-2 et MINI). En outre, les entretiens qualitatifs ont fait ressortir que les jeunes contrevenants pris en charge se trouvent à une croisée des chemins où s'affrontent le désir de quitter un mode de fonctionnement délinquant et le sentiment de ne pas pouvoir le faire à cause d'un avenir considéré comme étant bloqué. Dans la discussion, les résultats des devis quantitatifs et qualitatifs sont mis en relation, dans une démarche abductive, pour en proposer une compréhension intégrée. Finalement, quelques suggestions pour l'intervention complètent cet article. 


\title{
Jeunes contrevenants à la croisée des chemins
}

\section{Étude à devis mixte du risque suicidaire}

\author{
Catherine Laurier ${ }^{1}$ \\ Professeure adjointe \\ Département de psychoéducation, Université de Sherbrooke \\ Catherine.Laurier@usherbrooke.ca
}

Anne-Marie Ducharme

Candidate au doctorat en psychoéducation

Université de Sherbrooke

anne-marie.ducharme2@usherbrooke.ca

\section{Laurie St-Pierre}

Candidate au doctorat

Département de psychologie, Université de Sherbrooke

Laurie.morrisseau-st-pierre@usherbrooke.ca

\section{Janet Sarmiento}

Agente de planification, de programmation et de recherche Institut universitaire Jeunes en difficulté

Centre intégré universitaire de santé et de services sociaux du Centre-Sud-de-l'île-de-Montréal

Janet.Sarmiento@cjm-iu.qc.ca

RÉSUMÉ - En raison de la prédominance des conduites antisociales des jeunes contrevenants en général et de ceux associés aux gangs de rue en particulier, qui constituent d'ailleurs le motif de leur prise en charge par le système de justice, il est moins fréquent de se pencher sur leur victimisation et leurs séquelles psychologiques. Ainsi, dans le cadre de cet article, 212 jeunes contrevenants, pris en charge par les services jeunesse ou les services correctionnels ont été évalués à l'aide de questionnaires quantitatifs et lors d'une entrevue qualitative semi-structurée. Cette étude à devis mixte a permis de relever, dans la partie quantitative, les variables qui contribuent statistiquement le plus au risque suicidaire, tel qu'il a été évalué à l'aide de deux outils (MAYSI-2 et MINI). En outre, les entretiens qualitatifs ont fait ressortir que les jeunes contrevenants pris en charge se trouvent à une croisée des chemins où s'affrontent le désir de quitter un

1. Faculté d'éducation, Université de Sherbrooke, 2500, boul. de l'Université, Sherbrooke (Québec), Canada, J1K 2R1.

Criminologie, vol. 51, n 2 (2018) 
mode de fonctionnement délinquant et le sentiment de ne pas pouvoir le faire à cause d'un avenir considéré comme étant bloqué. Dans la discussion, les résultats des devis quantitatifs et qualitatifs sont mis en relation, dans une démarche abductive, pour en proposer une compréhension intégrée. Finalement, quelques suggestions pour l'intervention complètent cet article.

MoTS CLÉS - Jeunes contrevenants, risque suicidaire, méthode mixte, santé mentale, évaluation.

\section{Introduction}

Bien qu'il soit admis que la délinquance juvénile représente un problème en soi et par ses conséquences, notamment en ce qui a trait à la sécurité publique, elle provoque aussi son lot de problèmes concomitants chez les jeunes délinquants qui exigent attention. Les jeunes contrevenants s'exposent à une variété de troubles de santé mentale et présentent un risque suicidaire. Cet article porte sur les liens entre risque suicidaire et délinquance juvénile, examinés à l'aide d'un devis mixte alliant méthodes quantitative et qualitative. D'abord, les variables contribuant à la présence d'un risque suicidaire chez les jeunes contrevenants sont analysées par des analyses quantitatives. Les perceptions de ces jeunes quant à leur passage dans le système de justice et à leur avenir sont ensuite explorées à l'aide d'une analyse qualitative de type abductif, amenant des éléments de compréhension supplémentaires sur le développement des comportements suicidaires. Finalement, la mise en perspective des résultats issus du quantitatif et du qualitatif permet une discussion sur les éléments à considérer afin de mieux appréhender le risque suicidaire et la complexité de ce phénomène chez des jeunes contrevenants pris en charge. La démarche abductive permet de considérer plusieurs explications pouvant contribuer au risque suicidaire; celles-ci sont examinées plus en détail afin de ne retenir que les hypothèses qui semblent contribuer aux meilleures interprétations des données (Charmaz, 2006). Dans le cas de la présente recherche, les résultats quantitatifs seront éclairés par les résultats qualitatifs.

L'entrée légale dans le monde adulte à 18 ans ne signifie pas pour autant la fin des problèmes typiques de l'adolescence. Par exemple, pour l'Organisation des Nations Unies, la jeunesse concerne les personnes âgées de 15 à 24 ans (www.unesco.org). D’ailleurs, au Québec, les établissements jeunesse poursuivent leur mission de réadaptation des jeunes contrevenants ayant commis leur délit avant 18 ans jusqu'à l'atteinte de leurs 21 ans, alors que ceux qui commettent un délit à partir de l'âge de 18 ans sont pris en charge par les services correctionnels. 
Au Québec, en 2016, 10907 jeunes contrevenants ont reçu des services des centres jeunesse (Institut national d'excellence en santé et en services sociaux, 2016). De ce nombre, 430 mises sous garde ont été ordonnées et 4317 peines ont été purgées dans la collectivité.

Les services correctionnels assument les services pour les adultes (à partir de 18 ans) contrevenants. Au Québec, en 2015-2016, les services correctionnels comptaient quotidiennement en moyenne 18670 contrevenants sous leur responsabilité, dont 5097 détenus, ce qui représente 43165 nouvelles admissions en détention dans l'année (Reitano, 2017). La majorité des admissions provinciales (58\%) concernent les jeunes adultes (moins de 35 ans) (Reitano, 2017).

Les services jeunesse et les services correctionnels du Québec ont tous deux le mandat d'offrir des services aux personnes reconnues coupables d'un délit. Toutefois, les difficultés adaptatives et les troubles intériorisés ne font pas partie des motifs pour lesquels ces établissements en ont la responsabilité. Or, ces jeunes sont nombreux à présenter des troubles de santé mentale (McReynolds et al., 2008; Radeloff et al., 2015; Schwalbe, Gearing, Mackenzie, Brewer et Ibrahim, 2013; Soni, 2010; Wasserman, McReynolds, Schwalbe, Keating et Jones, 2010; Williams, Grisso, Valentine et Remsburg, 2008).

Tout comme pour les adultes, les études montrent que la psychopathologie est fortement associée aux idéations suicidaires, tentatives de suicide et suicides complétés (Laurier et Chagnon, 2011b), tant pour les troubles intériorisés (dépression et anxiété) que pour les troubles extériorisés (agressivité et délinquance). Des recensions d'écrits portant sur les facteurs de risque (Laurier et Chagnon, 2011b; St-Pierre et Laurier, 2018) et des recherches empiriques (Laurier et Chagnon, 2011a) étudiant ce phénomène ont pu mettre de l'avant que les jeunes contrevenants représentent une population particulièrement à risque de conduites suicidaires. En effet, ils seraient de 3 à 18 fois plus à risque de commettre une tentative de suicide que les jeunes de la population générale (Casiano, Katz, Globerman et Sareen, 2013). De 10 à $24 \%$ des jeunes contrevenants auraient eu des idées suicidaires au cours de l'année précédant la collecte de données (Abram et al., 2008; Bhatta, Jefferis, Kavadas, Alemagno et Shaffer-King, 2014; Chapman et Ford, 2008; Gretton et Clift, 2011 ; Kenny, Lennings et Munn, 2008; Moore, Gaskin et Indig, 2015; Schwalbe et al., 2013; Sedlak et McPherson, 2010; Suk et al., 2009; Williams et al., 2008) alors qu'entre $8,4 \%$ et $22 \%$ de ceux-ci auraient fait une tentative de suicide au cours de leur 
vie (Abram et al., 2008; Bhatta et al., 2014; Kenny et al., 2008; Kiriakidis, 2008; Moore et al., 2015; Sedlak et McPherson, 2010; Wasserman et al., 2010). Une étude québécoise de Laurier et Chagnon (2011a) estimait à $37 \%$ la proportion de jeunes contrevenants à risque de commettre une tentative de suicide. D'ailleurs, les données sur la mortalité au Québec pour la période 2010-2012 indiquent que près d'un tiers $(30,6 \%)$ des décès chez les jeunes hommes âgés de 15-24 ans étaient des suicides (Thibodeau et Perron, 2017).

Plusieurs facteurs pourraient contribuer à expliquer le risque suicidaire auquel s'exposent les jeunes contrevenants. Le modèle interactif de Laurier et Chagnon (2011b), développé à partir d'une revue de littérature, propose des explications au phénomène où les facteurs qui contribuent au risque suicidaire tendent à s'aggraver de façon réciproque. En effet, les événements de vie difficiles et stressants antérieurs aux comportements délinquants, en conjonction avec certains traits de personnalité (ex.: impulsivité, hostilité) et les comportements délinquants en eux-mêmes, créeraient une dynamique d'aggravation du risque suicidaire à travers le temps. Plus précisément, il y est proposé que les sanctions associées aux comportements délinquants provoquent l'isolement et un affaiblissement du réseau social chez certains, ce qui, jumelé à une santé mentale déjà précaire causée par les facteurs antérieurs à la délinquance (événements de vie, facteurs individuels, sociaux et résidentiels), entrainent une détérioration des conditions de vie et une augmentation de la vulnérabilité au risque suicidaire. En conséquence, ces jeunes seraient plus à risque de développer des psychopathologies comme la dépression, le sentiment de désespoir et l'anxiété quant à leur situation. Une recension des études portant sur les adolescents et les facteurs de risque de suicide indique en outre que ces trois troubles y sont fortement associés (Evans, Hawton et Rodham, 2004). En ce qui a trait aux événements stressants durant l'enfance, les abus sexuels et physiques sont reconnus comme des facteurs de risque suicidaire chez les jeunes contrevenants (Bhatta et al., 2014; Croysdale, Drerup, Bewsey et Hoffmann, 2008; Evans et al., 2004; Gretton et Clift, 2011; Hayes, 2009; Kenny et al., 2008; Laurier, 2008; Laurier et Chagnon, 2011b; National Action Alliance for Suicide Prevention: Youth in Contact with the Juvenile Justice System Task Force, 2013; Sedlak et McPherson, 2010; Stokes, McCoy, Abram, Byck et Teplin, 2015). Bien que moins documentés, les abus de nature émotionnelle, telles les remarques et les attaques verbales discréditant le sentiment de 
valeur personnelle de l'enfant ou son bien-être, ou encore les remarques humiliantes d'un adulte envers l'enfant (Bernstein et Fink, 1998), s'avèrent également liés au risque de suicide (Croysdale et al., 2008).

Croysdale et al. (2008) ont étudié l'association entre la victimisation, les troubles de santé mentale et le risque suicidaire. La victimisation sur le plan des maltraitances physiques, sexuelles et émotionnelles a été mesurée. Les jeunes révélant l'un ou l'autre des types de victimisation étaient deux fois plus nombreux à signaler avoir fait une tentative de suicide dans le passé, comparativement à ceux qui n'en faisaient aucune mention. De plus, les garçons qui mentionnent des abus de nature émotionnelle étaient significativement plus à risque de présenter un trouble de santé mentale et des comportements suicidaires.

Parmi les facteurs de risque inhérents aux comportements suicidaires ressort le mode de vie délinquant qui expose régulièrement les jeunes contrevenants à des situations où prévalent la violence et l'utilisation d'armes (Laurier, Guay, Lafortune et Toupin, 2015). Le port d'armes, bien souvent considéré comme nécessaire dans un mode de vie délinquant, fait en sorte que ces jeunes ont accès à des moyens létaux, augmentant encore le risque de suicides complétés (Laurier et Chagnon, 2011b). Le fait d'avoir commis des crimes violents est proposé comme un facteur de risque du suicide chez les jeunes contrevenants (Kiriakidis, 2008). Une comparaison des comportements à risque entre les jeunes itinérants de Los Angeles étant ou ayant été membres ou affiliés à un gang de rue et d'autres n'ayant aucun lien avec un gang de rue (Petering, 2016) indique que les jeunes membres d'un gang sont significativement plus à risque de faire une tentative de suicide. Ces jeunes présentaient des taux jusqu'à six fois plus élevés de violence interpersonnelle. De plus, même en tenant compte du niveau de délinquance ainsi que du fait d'être témoin de violence, les membres de gangs de rue seraient près de deux fois plus à risque que les non-membres d'avoir des comportements suicidaires. Outre leur niveau de délinquance plus élevé, les membres de gangs de rue présentent donc des caractéristiques les rendant plus vulnérables à des comportements suicidaires (Madan, Mrug et Windle, 2011). Selon Burton, Foy, Bwanausi, Johnson et Moore (1994) et Taylor, Peterson, Esbensen et Freng (2007), plus un individu utilise la violence, plus il serait à risque d'en être lui-même victime. L'étude de Viljoen, O’Neil et Sidhu (2005), menée en ColombieBritannique, dans un contexte légal s'apparentant à celui du Québec, a porté sur le phénomène de l'intimidation auprès de jeunes contreve- 
nants. Les taux de risque suicidaire étaient plus élevés tant chez les jeunes se disant uniquement victimes que chez ceux qui se disaient à la fois victimes et auteurs d'actes d'intimidation, comparativement à leurs homologues se décrivant uniquement comme auteurs d'actes d'intimidation. En raison des situations criminogènes dans lesquelles ils évoluent, les jeunes contrevenants sont plus fréquemment victimes de violence sévère que les autres adolescents et jeunes adultes (Chen, 2009). En outre, par leurs conduites antisociales, les jeunes contrevenants s'exposent à des conséquences néfastes pour eux-mêmes, à la fois pour leur développement social, physique et psychologique.

Une exposition répétée à des conditions de vie difficiles qui laisse des traces

Le fait que les jeunes contrevenants aient été souvent exposés dès un jeune âge à la violence et à des conditions de vie adverses pourrait induire chez eux un désir de s'en sortir. Certains peuvent valoriser des moyens adaptés socialement pour s'extraire de l'adversité dans laquelle ils ont jusqu'alors évolué, alors que d'autres peuvent au contraire se sentir impuissants et ne pas entrevoir d'issues (Ferrer et al., 2010; Flannery, Singer et Wester, 2003; Heath et Priest, 2016; Kim, Kim et Samuels-Dennis, 2012; Speckens et Hawton, 2005). Dans une comparaison entre un groupe de jeunes contrevenants et un groupe de jeunes non contrevenants, Ferrer et al. (2010) notent que les premiers adoptent des stratégies d'adaptation beaucoup moins efficaces lorsqu'ils font face à des situations difficiles. Ils ont tendance à se blâmer et à espérer résoudre de façon positive leurs ennuis, tout en ayant de faibles capacités personnelles pour résoudre les situations problématiques. Ils ont tendance à éviter de parler de leurs problèmes avec les autres et à s'isoler. Ces jeunes semblent se soucier de leur avenir, ce qui rejoint une préoccupation constante pour la jeunesse, peu importe les époques et les situations (Gratton et Lazure, 1996). La prise en charge par les services jeunesse et l'hébergement en centre de réadaptation peuvent offrir une nouvelle voie qui dessine d'autres perspectives ou, au contraire, confirmer un chemin déjà tracé d'avance, accentuant une perte d'espoir pouvant occasionner des idées suicidaires. Le modèle Cry of Pain, adapté à la réalité des jeunes contrevenants (St-Pierre et Laurier, 2018, ce numéro), présente le développement du risque suicidaire chez les jeunes contrevenants sous forme de trajectoire où s'enchaînent un 
sentiment de défaite lors de la première arrestation, une perception d'être pris au piège lors de la mise sous garde ainsi qu'un sentiment de désespoir. Le suicide devient alors une option pour échapper à cette situation pour certains qui cumulent des facteurs de risque à la fois personnels et institutionnels (St-Pierre et Laurier, 2018).

\section{Méthodologie}

La présente étude utilise un devis mixte, combinant méthodes quantitative et qualitative. Le volet quantitatif a permis d'examiner les variables sousjacentes au risque suicidaire chez les jeunes contrevenants afin d'y inclure celles qui étaient significatives dans un modèle de régression. Des questionnaires autorapportés ont été utilisés afin de mesurer le risque suicidaire, la délinquance, la santé mentale et les traumatismes vécus. La partie qualitative a été menée auprès d'un sous-groupe de l'échantillon et a exploré les thèmes entourant les comportements de prise de risque et les traumatismes liés aux comportements délinquants. L'utilisation de la méthode qualitative permet d'explorer plus en profondeur et de raffiner les résultats obtenus par la méthode quantitative, ajoutant des éléments que la première méthode ne pourrait saisir. La combinaison des deux méthodes permet donc de mieux comprendre dans son ensemble le phénomène étudié (Marpsat, 1999). Dans le cadre de cet article, l'ajout de la méthode qualitative à la suite des analyses quantitatives ouvre une nouvelle perspective et permet d'en bonifier l'interprétation.

\section{Volet quantitatif}

\section{Participants}

La présente étude a été menée de 2011 à 2013 auprès de 212 jeunes contrevenants de sexe masculin, âgés de 14 à 25 ans (moyenne 18,3 ans). L'échantillon total est composé de 155 (73\%) jeunes contrevenants sous la responsabilité des centres jeunesse du Québec et de 57 $(27 \%)$ sous la responsabilité de la Direction générale des services correctionnels (DGSC). La majorité des participants (83\%) se trouvait, au moment de l'étude, sous garde ou en détention alors que $17 \%$ étaient suivis dans la communauté. La durée prévue de leur prise en charge, lors de l'étude, variait entre 2 et 84 mois, la moyenne se situant à 21,6 mois. Parmi l'échantillon, 80 (39\%) ont autorévélé faire partie ou avoir déjà fait partie d'un gang de rue. 


\section{Procédure et instruments utilisés}

Les questionnaires ont été remplis par les participants ayant consenti à prendre part à la recherche. La passation de l'ensemble des questionnaires sur support informatique et l'entrevue structurée ont exigé deux rencontres d'en moyenne deux heures par participant lors desquelles un assistant de recherche était disponible pour soutenir les participants, répondre à leurs questions et expliquer les consignes. Dans le cadre de cet article, seules les variables à l'étude seront décrites et discutées.

Le risque suicidaire. Deux instruments ont été utilisés afin d'évaluer le risque suicidaire. Un premier, issu de la version française, et adaptée pour le Québec, du Massachusetts Youth Screening Questionnaire (MAYSI-2) (Grisso, Barnum, Fletcher, Cauffman et Peuschold, 2001) et un second, issu du Mini International Neuropsychiatric Interview (MINI). Le MAYSI-2 est un questionnaire autorapporté destiné spécifiquement aux jeunes contrevenants. Il est composé de 52 items auxquels les participants doivent répondre par oui ou par non, en regard des derniers mois. Il comprend 7 sous-échelles, dont les idéations suicidaires. Celles-ci sont mesurées par les réponses autorapportées à cinq items (détaillés au Tableau 1). Le score consiste en la somme des réponses affirmatives. Le MINI est une entrevue diagnostique structurée à partir des critères diagnostiques du DSM-IV-TR (American Psychiatric Association, 2000). Pour déterminer s'il y a présence ou non de risque chez les participants selon le MINI, six questions s'apparentant à celles du MAYSI-2 sont posées oralement aux participants, par l'assistant de recherche formé à l'outil.

La délinquance autorévélée. L'adaptation française du Self-Report of Offending-Revised (SRO-R) (Huizinga, Esbenson et Weiher, 1991) a été utilisée afin de mesurer la délinquance. Il s'agit d'un questionnaire autorévélé comportant 30 crimes. Les participants devaient indiquer s'ils avaient commis l'un ou l'autre de ceux-ci au cours des 12 mois précédant leur incarcération et, si oui, leur fréquence. Il s'agit d'un instrument validé et largement utilisé dans le cadre d'études portant sur les délinquants mineurs et adultes (Piquero, Farrington et Blumstein, 2003). Les paramètres de la délinquance ont été élaborés à partir des réponses au questionnaire. Quatre catégories de délinquance ont été formées (crimes contre la personne, crimes contre les biens, trafic de stupéfiants et autres infractions prévues au Code criminel). La diversité des délits commis a été mesurée selon les catégories de délits et leur fréquence correspond au nombre de délits commis durant les 12 mois précédant la prise en charge. 
La présence de traumatismes durant l'enfance. Un outil a été utilisé pour évaluer les traumatismes vécus durant l'enfance. Le Child Trauma Questionnaire (CTQ) (Bernstein et Fink, 1998), traduction française (Paquette, Laporte, Bigras et Zoccolillo, 2004), vise l'évaluation des traumatismes précoces (dans l'enfance). Il comporte 28 items divisés en 5 catégories (abus émotionnels, négligence émotionnelle, abus physiques et sexuels, négligence physique).

\section{Analyses}

Des analyses multivariées ont permis de constituer des modèles statistiques afin d'examiner les variables associées au risque suicidaire évalué par le MAYSI-2. D’abord, une régression linéaire multiple a été effectuée où la variable dépendante était le niveau de risque suicidaire (évalué par le MAYSI-2) et les variables indépendantes la délinquance autorévélée, les troubles de santé mentale évalués par le MINI et la présence de traumatismes. Ensuite, une seconde régression logistique a été effectuée avec les mêmes variables indépendantes, la variable dépendante étant cette fois le risque suicidaire tel qu'il a été évalué par le MINI.

\section{Volet qualitatif}

\section{Participants}

Les entrevues qualitatives ont été menées auprès d'un sous-groupe de 25 participants de l'échantillon total. Pour être sélectionnés, ils devaient être placés sous la responsabilité d'un centre jeunesse et présenter une association aux gangs de rue. La participation au volet qualitatif a été proposée aux premiers participants de la recherche qui correspondaient à ces critères, jusqu'à l'atteinte d'un échantillon de 25 jeunes contrevenants. Les jeunes ayant pris part à l'entretien qualitatif étaient âgés de 15 à 19 ans (moyenne 17,6 ans). Près de deux tiers de ces jeunes provenaient de quartiers défavorisés ou de familles criminalisées, côtoyant dès la préadolescence des membres de gangs et étant témoins d'actes de violence et de criminalité.

\section{Guide d'entretien}

Les entrevues semi-directives ont été réalisées conformément à un guide d'entretien. Les thèmes explorés étaient reliés à la participation à un 
gang de rue, à la prise de risque et aux traumas subis ainsi qu'à perception de ceux-ci, à la peur de mourir et au désir de vivre, aux modèles sociaux et finalement aux rêves concernant l'avenir. Pour chacun des thèmes, des sous-thèmes et des relances étaient prévus, afin d'approfondir certains sujets.

\section{Déroulement des entrevues}

Les entrevues ont été menées individuellement dans une salle assurant la confidentialité par une étudiante au doctorat en psychologie, en fin de parcours, qui a été formée par la chercheure principale de l'étude. La durée des entrevues a varié de 38 à 133 minutes, selon la capacité d'élaboration verbale du participant. Les rencontres ont eu lieu dans les bureaux du centre d'hébergement ou de réadaptation. Elles ont été enregistrées et retranscrites intégralement pour en faire l'analyse.

\section{Analyses}

Utilisant une approche abductive (Charmaz, 2006), qui s'inspire des fondements de l'approche de la théorisation ancrée (Glaser et Strauss, 1967; Paillé, 1994; Strauss et Corbin, 1990), et à l'aide du logiciel NVivo v10, des unités de signification (extraits) ont d'abord été définies pour faire émerger ensuite des catégories conceptuelles. L'analyse croisée contextualisée (environnement sociofamilial, expériences traumatiques, passé délinquant) a éclairé et raffiné l'interprétation du sens donné par les jeunes interviewés à leur vécu et à la perception de leur avenir.

\section{Résultats}

Analyses descriptives

Parmi l'échantillon, $22 \%(n=46)$ des jeunes contrevenants présentaient un risque suicidaire, selon les critères du MINI. Au MAYSI-2, près de $28 \%(n=59)$ des jeunes contrevenants avaient coché au moins une fois «oui» à l'une des cinq questions évaluant les idéations suicidaires telles que détaillées au Tableau 1.

\section{Délinquance autorévélée}

En moyenne, les participants ont révélé avoir commis leur premier délit à l'âge de 14 ans et rapporté en moyenne environ cinq différents types de délits $(5,2)$ dans les 12 derniers mois. Suivant l'ordre de fréquence 
TABLEA U 1

Statistiques descriptives du risque suicidaire évalué par le MINI et le MAYSI-2

\begin{tabular}{|llc|}
\hline Présence du risque suicidaire (MINI) & N & $\%$ \\
\hline Oui & 46 & 21,8 \\
Non & 165 & 78,2 \\
Total & $\mathbf{2 1 1}$ & $\mathbf{1 0 0}$ \\
\hline Niveau de risque suicidaire (MAYSI-2) (Nombre de questions & & \\
répondues par «oui» par participant) & $\mathbf{N}$ & $\%$ \\
0 & 153 & 72,2 \\
1 & 36 & 17,0 \\
2 & 14 & 6,6 \\
3 & 5 & 2,4 \\
4 & 2 & 0,9 \\
5 & 2 & 0,9 \\
Moyenne & 0,46 & \\
Médiane & 0 & \\
Écart-type & 0,91 & \\
Total & $\mathbf{2 1 2}$ & \\
\hline Répartition des réponses « oui » aux questions du MAYSI-2 & & \\
Dans le dernier mois... & $\mathbf{N}$ & $\%$ \\
As-tu souhaité être mort? & 23 & 10,8 \\
As-tu eu l'impression que la vie ne valait pas le coup d'être vécue? & 28 & 13,2 \\
As-tu eu l'impression de te faire du mal? & 27 & 12,7 \\
As-tu eu envie de te suicider? & 11 & 5,2 \\
As-tu perdu tout espoir dans ton existence? & 8 & 3,8 \\
\hline
\end{tabular}

dans la catégorie des crimes contre la personne se trouvent les voies de fait $(69,8 \%, n=148)$, les menaces $(46,7 \%, n=99)$, les vols qualifiés (33\%, $n=70)$, les décharges d'armes à feu $(20,3 \%, n=43)$ ainsi que les agressions sexuelles $(3,3 \%, n=7)$. Dans la catégorie des crimes contre les biens, dans l'ordre de fréquence se trouvent la fraude $(66,5 \%$, $n=141)$, les introductions par effraction $(40,6 \%, n=86)$, les méfaits $(29,2 \%, n=62)$ ainsi que les incendies criminels $(15,6 \%, n=33)$. En outre, $62,3 \%(n=132)$ rapportent le trafic de stupéfiants et $72,2 \%$ $(n=153)$ des délits d'un autre type que ceux énumérés. Ils présentent une moyenne de 1,2 crime sans violence et de 2,3 crimes avec violence au cours des 12 mois précédant leur incarcération. 
Les traumatismes vécus durant l'enfance

Au CTQ, les jeunes contrevenants rencontrés présentent des scores moyens de 3,8 à l'échelle des abus émotionnels, 5,5 en ce qui a trait à la négligence émotionnelle, 3,8 en ce qui a trait aux abus physiques et 1,25 en lien avec les abus sexuels.

TABLEAU 2

Statistiques descriptives des variables continues

\begin{tabular}{|l|c|c|c|c|c|c|}
\hline & Moy. & Méd. & $\min$ & $\max$ & É-T \\
\hline Paramètres de la délinquance \\
\hline Diversité de la délinquance & 5,19 & 5,00 & 0 & 11 & 3,27 \\
\hline Fréquence crimes de violence (LOG) & 1,20 & 1,23 & 0,30 & 2,76 & 0,60 \\
\hline Fréquence crimes sans violence (LOG) & 2,32 & 2,54 & 0,30 & 5,24 & 0,92 \\
\hline Présence de traumatismes & 5,47 & 4,50 & 0 & 19 & 4,88 \\
\hline Négligence émotionnelle & 3,17 & 1,00 & 0 & 19 & 4,06 \\
\hline Abus physiques & 3,77 & 3,00 & 0 & 19 & 4,01 \\
\hline Abus émotionnels & 1,25 & 0,00 & 0 & 19 & 2,67 \\
\hline Abus sexuels
\end{tabular}

La santé mentale (MINI)

En ce qui a trait à la santé mentale des participants telle que révélée par le MINI, $18,2 \%(n=38)$ vivaient un épisode dépressif et 7,2\% $(n=15)$ un épisode maniaque, $1,4 \%(n=3)$ souffraient d'agoraphobie, $5,7 \%$ $(n=12)$ présentaient un trouble panique, $1,5 \%(n=3)$ une agoraphobie avec trouble panique, $1,9 \%(n=4)$ un trouble de phobie sociale, $0,5 \%(n=1)$ un trouble obsessionnel compulsif, $22,5 \%(n=47)$ un trouble d'anxiété généralisée et $27,7 \%(n=57)$ un état de stress posttraumatique.

\section{Analyses de régression}

Un premier modèle a été réalisé en effectuant une régression linéaire multiple afin de déterminer les variables permettant d'expliquer statistiquement la variation du niveau de risque suicidaire $(F=24,43$; $\mathrm{p}<0,001)$, tel qu'il a été évalué par le MAYSI-2. Les résultats sont présentés au Tableau 4. Labus émotionnel durant l'enfance apparaît comme la variable la plus fortement associée à un niveau de risque 
TA B LEA U 3

Fréquences des critères diagnostiques du MINI

\begin{tabular}{|l|c|}
\hline & $\%$ \\
\hline Épisode dépressif & 18,2 \\
\hline Épisode maniaque & 7,2 \\
\hline Agoraphobie & 1,4 \\
\hline Trouble panique & 5,7 \\
\hline Agoraphobie avec trouble panique & 1,5 \\
\hline Phobie sociale & 1,9 \\
\hline Trouble obsessionnel compulsif & 0,5 \\
\hline Anxiété généralisée & 22,5 \\
\hline État de stress post-traumatique & 27,7 \\
\hline Utilisation nocive de l'alcool & 19,8 \\
\hline Utilisation nocive de drogues & 25,0 \\
\hline
\end{tabular}

TA B LEA U 4

Régression linéaire de la présence du risque suicidaire, tel qu'il a été évalué par le MAYSI-2

\begin{tabular}{|l|c|c|c|}
\hline & B & ET B & Bêta \\
\hline Traumatismes durant l'enfance & - & - & - \\
\hline Abus émotionnel & $0,06^{* *}$ & 0,01 & 0,27 \\
\hline MINI & - & - & - \\
\hline Épisode dépressif & $0,61^{* *}$ & 0,15 & 0,26 \\
\hline Trouble panique & $0,88^{* *}$ & 0,24 & 0,22 \\
\hline CONSTANTE & 0,06 & 0,08 & - \\
\hline $\mathrm{R}^{2}$ total & & 0,26 & \\
\hline $\mathrm{F}$ & & $24,43^{* *}$ & \\
\hline$n$ & & 212 & \\
\hline
\end{tabular}

Note: ${ }^{* *} p<0,001$.

suicidaire plus élevé $(\beta=0,27 ; p<0,001)$. Le fait de vivre un épisode dépressif $(\beta=0,26 ; p<0,001)$ ainsi que le fait de vivre avec un trouble panique $(\beta=0,22 ; p<0,001)$ se révèlent aussi prédicteurs du risque suicidaire, dans une moindre mesure. Ainsi, les jeunes contrevenants qui révèlent avoir été victimes d'abus émotionnels dans l'enfance et ceux qui répondent aux critères pour un épisode dépressif ou pour le trouble 
panique présentent un niveau de risque suicidaire significativement plus élevé, tel qu'il a été évalué par le MAYSI-2.

Un deuxième modèle a été réalisé en faisant une régression logistique. Les résultats sont présentés au Tableau 5. Afin d'expliquer la présence ou non d'un risque suicidaire chez les jeunes contrevenants, les variables à l'étude les plus pertinentes (soit celles où il y avait une différence significative en comparant les adolescents présentant ou non un risque suicidaire) ont été intégrées dans un même modèle de régression $\left(\mathrm{X}^{2}=40,85 ; \mathrm{p}<0,001\right)$. Les résultats indiquent que parmi les variables étudiées, seules les expériences d'abus émotionnels $(b=0,20$; $\mathrm{p}<0,001)$ et d'abus sexuels $(b=0,12 ; \mathrm{p}<0,05)$ durant l'enfance sont associées significativement à la présence d'un risque suicidaire. Il ressort donc qu'en considérant toutes les autres variables selon le MINI, les jeunes révélant avoir été victimes d'abus émotionnels dans l'enfance sont $22 \%$ plus susceptibles de présenter un risque suicidaire (zone à risque ou danger) alors que ceux qui révèlent avoir été victimes d'un abus sexuel sont $13 \%$ plus à risque.

\section{Analyses qualitatives}

Le centre jeunesse à la croisée des chemins

Lors des entrevues qualitatives, les jeunes ont été amenés à parler de leur situation de prise en charge par les services jeunesse. Il s'en dégage

TABLEAU 5

Régression logistique de la présence du risque suicidaire, tel qu'il a été évalué par le MINI

\begin{tabular}{|l|c|c|c|c|}
\hline & B & $E T$ B & Wald & Exb (B) \\
\hline Traumatismes durant l'enfance & - & - & - & - \\
\hline Abus émotionnels & $0,20^{* *}$ & 0,05 & 17,60 & 1,22 \\
\hline Abus sexuels & $0,12^{*}$ & 0,06 & 4,01 & 1,13 \\
\hline MINI & - & - & - & - \\
\hline Trouble panique & 1,15 & 0,69 & 2,74 & 3,15 \\
\hline Épisode dépressif & 0,77 & 0,44 & 3,12 & 2,16 \\
\hline CONSTANTE & $-2,66^{* *}$ & 0,33 & 63,31 & 0,07 \\
\hline$R^{2}$ de Nagelkerke & \multicolumn{3}{|c|}{0,27} & \\
\hline Khi-deux & \multicolumn{5}{|c|}{212} \\
\hline$n$ & \multicolumn{5}{|c|}{} \\
\hline
\end{tabular}

Note: ${ }^{*} \mathrm{p}<0,05 ;{ }^{* *} \mathrm{p}<0,001$. 
une nette dichotomie entre les sentiments éprouvés à l'égard des aspects physiques et matériels liés à l'ordonnance de placement et les sentiments quant aux interventions effectuées dans le contexte de leur prise en charge. Dans un cas comme dans l'autre, ces sentiments peuvent être négatifs ou positifs, mais ils nous renseignent sur la façon dont l'ordonnance de placement est vécue par les jeunes contrevenants.

En ce qui concerne l'intervention dont ils ont été l'objet, plusieurs soulignent avoir été amenés à réfléchir au sujet de leurs comportements, des conséquences de leurs délits et des risques pris dans la vie. Comme Pascal l'exprime: «On réfléchit beaucoup, ce n'est pas facile être ici, tu es enfermé, tu y penses après ça, ça sert à quoi de faire ça? C'est ça, le centre jeunesse a sauvé ma vie. Le centre jeunesse a sauvé ma vie parce que je n'aurais jamais arrêté.»

La prise en charge par les centres jeunesse peut par ailleurs représenter pour plusieurs jeunes un sentiment d'échec puisqu'ils ont été arrêtés dans leurs conduites délinquantes et se sont littéralement fait prendre à la suite d'un délit. Ils peuvent en outre ressentir un sentiment de perte de liberté. La mise sous garde signifie par ailleurs la perte des liens avec ceux qu'ils considéraient jusqu'alors comme leur "vraie» famille (leurs pairs délinquants); c'est aussi une prise de conscience de la peine qu'ils peuvent avoir causée à leurs parents. Un jeune souligne que le fait d'avoir été hébergé lui a permis de prendre conscience de l'importance de sa famille dans sa vie; il raconte que sa mère est venue lui rendre visite et non ceux qu'il considérait comme ses amis. Philippe raconte que son expérience à la cour lui a permis de constater l'importance de sa famille pour lui: «Ma mère pleurait, ma grand-mère pleurait, mon petit frère pleurait. C'est fou. C'est ça qui m'a touché le plus. C'est ça qui m'a fait réaliser que j'avais une famille [...].» En outre, cette prise de conscience peut amorcer une réflexion sur les impacts des délits et faire naître une détresse qui n'était pas ressentie de façon aussi prégnante jusqu'alors, comme en témoignent les propos de Charles: "Quand tu vois tes parents pleurer à la Cour puis tout, tu n'as pas juste fait mal à tes victimes. Tu as fait mal à tout le monde autour de toi.»

La réflexion des adolescents rencontrés porte aussi sur leurs perspectives d'avenir; le dilemme entre continuer les activités délinquantes ou prendre la voie du désistement (retour à l'école, trouver un emploi légal, s'éloigner des pairs délinquants et, incluant dans certains cas, quitter leur quartier) est souvent déchirant et peut être source d'appréhension. Ils peuvent ressentir que la lourdeur de leur passé criminel peut avoir hypothéqué leur avenir. 
Plus tu es longtemps délinquant, moins tu as d'options après parce que les portes se ferment. Puis tu as 18 ans, tu peux avoir un dossier criminel qui vient de se fermer. Tu es rendu plus vieux, tu ne peux même plus aller à certaines places pour étudier... C'est d'autres portes qui se ferment. C'est vraiment plus tu restes longtemps, moins tu as de chances de changer. (Gino)

Devant les efforts requis pour entreprendre un désistement, combiné à la crainte de l'échec, le désespoir ne laisse parfois entrevoir que la mort comme dernière porte de sortie, comme Gino le souligne par ses propos.

C'est l'un ou l'autre (...). Je ne serai pas dans un bloc-appartements avec un chèque de bien-être social, puis je ne serai pas au centre-ville en train de quêter de l'argent. C'est soit que je vis bien, soit que je suis au pénitencier ou je suis mort. C'est comme ça que je vois... c'est soit que tu vis, soit que tu ne vis pas. Je ne veux pas survivre.

\section{Détresse associée à la délinquance traumatique}

La presque totalité des jeunes ont parlé de leur détresse directement lors des entretiens. Cette détresse peut être liée à des situations traumatiques vécues par les jeunes contrevenants. Les propos de MarcAndré, qui raconte le décès d'un ami lors d'un délit, l'illustrent:

Oui j'étais triste, j'étais très triste. Mais aussi je devenais de plus en plus agressif quand les gens disaient des trucs comme ça. Quand quelqu'un sortait une arme, je devenais de plus en plus agressif. En même temps, j'avais peur (...), mais en même temps, ça me fâchait comme...

Les propos de Marc-André témoignent de la tristesse et de l'agressivité en réaction à des situations de délinquance. Au-delà de l'agressivité en réaction aux situations difficiles vécues lors de délits, les jeunes expriment de la peur, voire de l'anxiété. Chris parle même de la difficulté à vivre avec un geste très grave qu'il a commis:

J'y pense toujours. [...] Je me remémore la journée que c'est arrivé [...] dans ma tête puis je fais quelque chose de spécial tout le temps, parce que ça fait deux ans que c'est arrivé puis à chaque année je fais quelque chose de spécial en son honneur. L'année passée, j'ai duré une semaine sans parler à personne. Je n'ai pas dit un mot de la semaine puis [...]. [Je me sens] impuissant. Je ne peux rien faire à ça, ça va toujours exister. Je ne pourrai pas l'oublier, à moins que je devienne super vieux puis je fais de l'Alzheimer, je ne pourrai pas oublier ce genre d'affaires là. Mais j'essaie d'apprendre à vivre avec ça. 


\section{Discussion}

Les analyses statistiques réalisées dans le cadre de cette étude ont permis de mettre de l'avant le fait que le vécu traumatique des jeunes est déterminant pour comprendre et mieux appréhender leur risque suicidaire. Peu importe l'outil utilisé pour évaluer ce risque (MINI ou MAYSI-2), les jeunes contrevenants qui ont révélé avoir été victimes d'abus émotionnels dans l'enfance apparaissent plus à risque que les autres. Par ailleurs, les entretiens qualitatifs réalisés avec un sous-échantillon ne semblent pas, à première vue, confirmer cette piste. Cette divergence entre ce qui est autorévélé par les questionnaires et ce qui est discuté en entrevue face à face semble révéler une difficulté éprouvée par les jeunes contrevenants lorsqu'ils perçoivent devoir exposer leur vulnérabilité. Alors que le lien entre les abus sexuels et physiques vécus et les comportements suicidaires a été démontré auparavant (Bhatta et al., 2014; Croysdale et al., 2008; Evans et al., 2004; Gretton et Clift, 2011; Hayes, 2009; Kenny et al., 2008; Laurier, 2008; National Action Alliance for Suicide Prevention: Youth in Contact with the Juvenile Justice System Task Force, 2013; Sedlak et McPherson, 2010; Stokes et al., 2015), le lien entre les abus émotionnels et les comportements suicidaires est resté, jusqu'à maintenant, peu étudié. Dans le cadre de la présente étude, les résultats dégagés sur la base du MINI portent à croire que l'expérience d'abus émotionnels s'avère un élément important dans la compréhension du risque suicidaire, tout comme les expériences d'abus sexuels. Ils rappellent ceux de Croysdale et al. (2008) ayant exploré le lien entre différentes formes d'abus (émotionnels, physiques et sexuels) et le suicide: la variable abus émotionnel s'étant aussi avérée significativement liée à l'historique suicidaire. Or, il importe aussi de souligner que le contexte même de la présente recherche, soit auprès de jeunes contrevenants pris en charge par les services correctionnels ou les centres jeunesse, a pu contribuer à ce que la maltraitance émotionnelle et sexuelle apparaisse comme des facteurs associés au risque suicidaire. En effet, la recherche de Lafortune et al. (2015) a révélé que $57,5 \%$ des jeunes contrevenants auraient connu une expérience de prise en charge en protection de la jeunesse en raison de maltraitance.

De même, dans la présente étude, toutes choses étant égales par ailleurs, il apparaît que le fait de vivre un épisode dépressif majeur ou un trouble panique fait augmenter le risque suicidaire chez ces jeunes. Le lien entre la dépression et les comportements suicidaires est largement reconnu dans le milieu scientifique (Abram et al., 2008; Bhatta et 
al., 2014; Chapman et Ford, 2008; Evans et al., 2004; Gretton et Clift, 2011; Schwalbe et al., 2013; Sedlak et McPherson, 2010; Suk et al., 2009; Wasserman et al., 2010). Toutefois, le trouble panique a fait l'objet de moins d'études concernant son association avec le risque suicidaire. Néanmoins, les résultats des études s'étant intéressées aux troubles anxieux (Abram et al., 2008; Kenny et al., 2008; McReynolds et al., 2008; Schwalbe et al., 2013; Sedlak et McPherson, 2010; Suk et al., 2009; Wasserman et al., 2010) et au risque suicidaire vont dans le même sens.

Il est dès lors possible de comprendre le risque suicidaire des jeunes contrevenants selon une perspective développementale dans laquelle des abus émotionnels et sexuels dans l'enfance viennent affecter l'adaptation des jeunes contrevenants. Les jeunes qui étaient déprimés et ceux qui affichaient un trouble panique présentaient un risque suicidaire accru. Il est possible d'interpréter que voir son avenir de façon sombre ou être en proie à des peurs peut exacerber les pensées suicidaires. L'analyse qualitative éclaire ces caractéristiques et permet de cerner un peu mieux comment a pu se développer le risque suicidaire chez ces jeunes contrevenants. Ceux qui ont été rencontrés ont fait part de la difficulté qu'ils pouvaient éprouver à vivre avec le souvenir de certains événements reliés à leurs activités délinquantes, comme la mort d'un ami ou un acte de violence qu'ils auraient commis. La présente étude vient appuyer l'idée qu'un mode de vie délinquant, marqué par la violence, peut engendrer de nouveaux traumatismes et miner la santé mentale des jeunes contrevenants, amplifiant le risque suicidaire (Laurier et al., 2015; Madan et al., 2011).

Les événements de délinquance vécus, mais surtout leurs conséquences, causent une détresse marquée chez les jeunes qui tentent de se projeter dans l'avenir. Les adolescents rencontrés semblent à la fois avoir le désir de quitter l'univers de délinquance dans lequel ils évoluent et parallèlement avoir le sentiment que leur avenir est bloqué. Comme l'a souligné Shelton (2004), les jeunes contrevenants peuvent avoir l'impression qu'ils manquent de contrôle sur leur vie, de soutien familial et communautaire. Ils expriment des sentiments de désespoir et de dépression, mais sont à la fois convaincus qu'il est nécessaire d'afficher une allure «dure» pour ne pas être atteints par la maladie mentale. Abrams et Aguilar (2005) rapportent que les jeunes contrevenants hébergés, malgré leur situation de délinquance, démontrent de l'espoir en l'avenir notamment grâce à la rencontre de modèles positifs. 
Or, la confiance en l'avenir serait un facteur de protection important contre le risque suicidaire. Ces jeunes contrevenants apparaissent par ailleurs à risque de suicide en raison de facteurs bien connus tels que l'accès aux armes, l'impulsivité et des moyens d'adaptation peu adéquats. La présente étude combinant méthodes quantitative et qualitative a permis de mettre en lumière un cheminement plus précis et de comprendre que ces adolescents, au passé difficile et présentant des liens probablement très fragiles avec leurs parents, sont dans un état où règne la peur, l'épisode panique pouvant être un facteur de risque supplémentaire. Par ailleurs, le trouble dépressif majeur est aussi lié à ce risque, comme il en est pour les autres groupes de personnes où le risque suicidaire a été évalué (Abram et al., 2008; Bhatta et al., 2014; Chapman et Ford, 2008; Gretton et Clift, 2011; Schwalbe et al., 2013; Sedlak et McPherson, 2010; Suk et al., 2009; Wasserman et al., 2010). Les analyses qualitatives présentées sommairement dans le cadre de cet article ont permis d'illustrer comment la prise en charge en centre jeunesse s'ajoute comme un élément pouvant exacerber la détresse associée à la maltraitance vécue dans l'enfance, mais pouvant aussi constituer une croisée des chemins vers un désir de changer de voie, de s'extraire de cet univers de délinquance.

\section{Conclusion}

L'ajout de l'abus émotionnel durant l'enfance, parmi les autres types d'abus plus fréquemment explorés dans les études précédentes, a permis de constater l'influence de ce type de traumatisme dans le développement $\mathrm{du}$ risque suicidaire chez les jeunes contrevenants. La présence d'abus émotionnels vécus durant l'enfance et autorévélée par l'adolescent s'est avérée le plus fort prédicteur - même devant les abus sexuels - du risque de suicide parmi les jeunes rencontrés, selon les analyses statistiques effectuées. Il importe cependant de garder à l'esprit que les participants à l'étude étaient des jeunes contrevenants pris en charge par les centres jeunesse ou les services correctionnels. Ces jeunes hommes sont plus susceptibles d'avoir vécu de la maltraitance dans l'enfance que d'autres provenant de la population générale. En effet, $57,5 \%$ des jeunes pris en charge en vertu de la Loi sur le système de justice pénale pour adolescents ont reçu des services sous la Loi de la protection de la jeunesse, selon Lafortune et al. (2015), témoignant de la forte prévalence des situations d'abus et de négligence dans leur 
passé. Les abus émotionnels et sexuels durant l'enfance font partie de l'équation pour comprendre le risque suicidaire chez les jeunes contrevenants; il est toutefois possible qu'il s'agisse d'un facteur de risque propre à cette population qui ne se retrouverait pas chez d'autres adolescents et jeunes adultes, non contrevenants.

Le fait de vivre un épisode dépressif ou un trouble panique augmente aussi le risque de présenter des idéations ou des conduites suicidaires chez les jeunes contrevenants. Alors que l'association entre dépression et risque suicidaire est bien connue et documentée, le trouble panique apparaît moins habituel. Au sein de la population contrevenante néanmoins, en conjonction avec les résultats issus de l'analyse qualitative et d'une démarche abductive, le trouble panique peut être compris comme un état où la peur transparaît dans l'ensemble du fonctionnement du jeune. Ce dernier peut se sentir pris au piège et ne pas pouvoir envisager une issue possible à la situation précaire empreinte de violence dans laquelle il évolue. Dans une optique de prévention, il importe de consacrer du temps à ces adolescents qui peuvent, bien qu'ils soient connus en raison des actes de violence qu'ils ont commis, en subir les contrecoups sur le plan de l'adaptation.

En conclusion, les jeunes contrevenants rencontrés dans le cadre de cette étude semblent coincés au sein d'une dynamique où s'affrontent le désir de s'en sortir (de la délinquance et des sentiments plus dépressifs), principalement pour ceux qui vont bien au moment de la recherche, et le sentiment de ne pas pouvoir y arriver. Ainsi, la prise en charge et l'ordonnance de placement en centre jeunesse peuvent être perçues comme salvatrices ou comme venant confirmer une situation dans laquelle ils se sentent confinés. La prise en charge a été vécue pour plusieurs comme une croisée des chemins.

À des fins d'intervention, il apparaîtra pertinent de bien cerner les antécédents de victimisation des jeunes contrevenants, en incluant l'abus émotionnel et sexuel, et ce, afin d'être en mesure d'évaluer le risque suicidaire. En plus d'être vigilant quant à la présence d'épisodes dépressifs majeurs, représentant un facteur de risque habituel, il importera en outre d'évaluer les symptômes liés au trouble panique et de laisser place à l'expression de leurs craintes et de leurs peurs. Dans une optique de réadaptation, il est tout à fait avisé de favoriser l'élaboration de plans d'avenir chez les jeunes contrevenants afin qu'ils puissent y trouver espoir et bâtir de nouvelles aspirations, alors qu'ils sont à cette croisée des chemins que représente la prise en charge en services jeunesse ou services correctionnels. 


\section{Références}

Abram, K. M., Choe, J. Y., Washburn, J. J., Teplin, L. A., King, D. C. et Dulcan, M. K. (2008). Suicidal ideation and behaviors among youths in juvenile

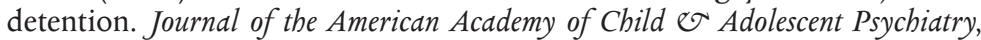
47(3), 291-300. https://doi.org/https://doi.org/10.1097/CHI. ob013e318160b3ce

Abrams, L. S. et Aguilar, J. P. (2005). Negative trends, possible selves, and behavior change: A qualitative study of juvenile offenders in residential treatment. Qualitative Social Work, 4(2), 175-196. https://doi.org/10.1177/ 1473325005052392

American Psychiatric Association. (2000). Diagnostic and statistical manual of mental disorders (DSM-IV-TR). Washington, DC: American Psychiatric Association.

Arnett, J. J. (1996). Sensation seeking, aggressiveness, and adolescent reckless behavior. Personality and Individual Differences, 20(6), 693-702. https://doi. org/10.1016/0191-8869(96)00027-X

Arnett, J. J. et Jensen, L. A. (1994). Socialization and risk behavior in two countries: Denmark and the United States. Youth of Society, 26(1), 3-22. https://doi.org/10.1177/0044118X94026001001

Bernstein, D. P. et Fink, L. (1998). Childhood trauma questionnaire: A retrospective self-report: Manual. Orlando, FL: Psychological Corporation.

Bhatta, M. P., Jefferis, E., Kavadas, A., Alemagno, S. A. et Shaffer-King, P. (2014). Suicidal behaviors among adolescents in juvenile detention: role of adverse life experiences. PLOS ONE, 9(2), e89408. https://doi.org/ 10.1371/journal.pone.0089408

Braunschweig, G., Francescotti, E., Schmid, M., Fegert, J. M. et Schmeck, K. (2010, septembre). The use of the MAYSI-2 in the three linguistic regions of Switzerland within the assessment of young people during penal or civil placement measures. Conférence présentée au 2e Congrès International de l'Association européenne de pédopsychiatrie légale, Basel, Suisse.

Burton, D., Foy, D., Bwanausi, C., Johnson, J. et Moore, L. (1994). The relationship between traumatic exposure, family dysfunction, and post-traumatic stress symptoms in male juvenile offenders. J Trauma Stress, 7(1), 83-93.

Casiano, H., Katz, L. Y., Globerman, D. et Sareen, J. (2013). Suicide and deliberate self-injurious behavior in juvenile correctional facilities: A review. Journal of the Canadian Academy of Child and Adolescent Psychiatry, 22(2), 118124.

Chapman, J. F. et Ford, J. D. (2008). Relationships between suicide risk, traumatic experiences, and substance use among juvenile detainees. Archives of Suicide Research, 12(1), 50-61. https://doi.org/10.1080/13811110701800830

Charmaz, K. (2006). Constructing grounded theory. A practical guide through qualitative analysis. Londres, Royaume-Uni: Sage Publications.

Chen, X. (2009). The link between juvenile offending and victimization. Youth Violence or Juvenile Justice, 7(2), 119-135.

Corff, Y. L. et Toupin, J. (2009). Comparing persistent juvenile delinquents and normative peers with the Five-Factor Model of Personality. Journal of 
Research in Personality, 43(6), 1105-1108. https://doi.org/10.1016/j. jrp.2009.06.011

Costa Jr., P. T. et McCrae, R. R. (1997). Stability and change in personality assessment: The Revised NEO Personality Inventory in the year 2000. Journal of Personality Assessment, 68(1), 86-94. https://doi.org/10.1207/ s15327752jpa6801_7

Croysdale, A. E., Drerup, L. C., Bewsey, K. et Hoffmann, N. G. (2008). Correlates of victimization in a juvenile justice population. Journal of Aggression, Maltreatment of Trauma, 17(1), 103-117. https://doi.org/10.1080/ 10926770802250876

Evans, E., Hawton, K. et Rodham, K. (2004). Factors associated with suicidal phenomena in adolescents: A systematic review of population-based studies. Clinical Psychology Review, 24(8), 957-979. https://doi.org/10.1016/j. cpr.2004.04.005

Ferrer, M., Carbonell, X., Sarrado, J. J., Cebrià, J., Virgili, C. et Castellana, M. (2010). Distinguishing male juvenile offenders through personality traits, coping strategies, feelings of guilt and level of anger. The Spanish Journal of Psychology, 13(2), 751-764. https://doi.org/10.1017/S1138741600002419

Flannery, D. J., Singer, M. I. et Wester, K. L. (2003). Violence, coping, and mental health in a community sample of adolescents. Violence and Victims, 18(4), 403-418. https://doi.org/10.1891/vivi.2003.18.4.403

Frydenberg, E. et Lewis, R. (1993). Adolescent coping scale. Melbourne, Australie: Australian Council for Educational Research.

Glaser, B. et Strauss, A. (1967). The discovery of grounded theory: Strategies for qualitative research. New Brunswick, NJ: Aldine.

Gratton, F. et Lazure, J. (1996). Les suicides d'être de jeunes québécois. Sainte-Foy, Québec: Presses de l'Université du Québec.

Gretton, H. M. et Clift, R. J. (2011). The mental health needs of incarcerated youth in British Columbia, Canada. International Journal of Law $\mathfrak{F}$ Psychiatry, 34(2), 109-15. https://doi.org/10.1016/j.ijlp.2011.02.004

Grisso, T., Barnum, R., Fletcher, K. E., Cauffman, E. et Peuschold, D. (2001). Massachusetts youth screening instrument for mental health needs of juvenile justice youths. Journal of the American Academy of Child $\mathcal{\sim}$ Adolescent Psychiatry, 40(5), 541-548.

Hayes, L. M. (2009). Juvenile suicide in confinement: A national survey. Washington, DC: U.S. Department of Justice.

Heath, R. A. et Priest, H. M. (2016). Examining experiences of transition, instability and coping for young offenders in the community: A qualitative analysis. Clinical Child Psychology and Psychiatry, 21(2), 224-239. https://doi. org/10.1177/1359104515581715

Huizinga, D., Esbenson, F. et Weiher, A. (1991). Are there multiple paths to delinquency? Journal of Criminal Law and Criminology, 82(1), 83-118.

Institut national d'excellence en santé et en services sociaux. (2016). Les mauvais traitements psychologiques: un mal silencieux (bilan des directeurs de la protection de la jeunesse/directeurs provinciaux 2016). Québec, Québec: Gouvernement du Québec. 
Kenny, D. T., Lennings, C. J. et Munn, O. A. (2008). Risk factors for self-harm and suicide in incarcerated young offenders: implications for policy and practice. Journal of Forensic Psychology Practice, 8(4), 358-382. https://doi. org/10.1080/15228930802199317

Kim, H.-S., Kim, H.-S. et Samuels-Dennis, J. (2012). The influence of psychosomatic symptoms, physical and sexual abuse, and coping strategies on delinquent behavior among Korean adolescents. Archives of Psychiatric Nursing, 26(2), 155-164. https://doi.org/10.1016/j.apnu.2011.06.006

Kiriakidis, S. P. (2008). Bullying and suicide attempts among adolescents kept in custody. Crisis, 29(4), 216-218.

Lafortune, D., Royer, M. N., Rossi, C., Turcotte, M. E., Boivin, R., Cousineau, M. M., ... Trépanier, J. (2015). La loi sur le système de justice pénale pour les adolescents sept ans plus tard: portrait des jeunes, des trajectoires et des pratiques (Rapport nº FQRSC 2011-TA-144097).

Laurier, C. (2008). Les facteurs associés aux comportements suicidaires chez les adolescents délinquants. Frontières, 21(1).

Laurier, C. et Chagnon, F. (2011a). Étude du risque suicidaire chez des jeunes contrevenants masculins: Caractéristiques différentielles des adolescents les plus à risque. Revue de Psychoéducation, 40(2), 191-216.

Laurier, C. et Chagnon, F. (2011b). Modèle interactif du risque suicidaire chez les jeunes contrevenants. Criminologie, 44(2), 251-278. https://doi.org/ 10.7202/1005799ar

Laurier, C., Guay, J.-P., Lafortune, D. et Toupin, J. (2015). Évaluer pour prévenir: les caractéristiques de la personnalité et les risques pris par les jeunes contrevenants associés aux gangs de rue (Rapport de recherche). Fonds de recherche Société et Culture Québec.

Leclerc, D., Pronovost, J. et Dumont, M. (2009). Échelle de coping pour adolescent: validation canadienne-française de l'adolescent coping scale de Frydenberg et Lewis (1993). Revue québécoise de psychologie, 30(1), 177-196.

Le Corff, Y. (2011). Role of personality traits in psychological treatment. Canadian Journal of Counselling and Psychotherapy/Revue canadienne de Counseling et de Psychothérapie, 45(3), 262-279.

Le Corff, Y. et Toupin, J. (2010). The five-factor model of personality at the facet level: association with antisocial personality disorder symptoms and prediction of antisocial behavior. Journal of Psychopathology and Behavioral Assessment, 32(4), 586-594. https://doi.org/10.1007/s10862-010-9180-y

Madan, A., Mrug, S. et Windle, M. (2011). Brief report: Do delinquency and community violence exposure explain internalizing problems in early adolescent gang members? Journal of Adolescence, 34(5), 1093-1096.

Marpsat, M. (1999). Les apports réciproques des méthodes quantitatives et qualitatives: le cas particulier des enquêtes sur les personnes sans domicile. Repéré à https:// www.ined.fr/fr/publications/document-travail/les-apports-reciproquesdes-methodes-quantitatives-et-qualitatives/

McReynolds, L. S., Wasserman, G. A., DeComo, R. E., John, R., Keating, J. M. et Nolen, S. (2008). Psychiatric disorder in a juvenile assessment center. Crime $\mathfrak{}$ Delinquency, 54(2), 313-334. 
Moore, E., Gaskin, C. et Indig, D. (2015). Attempted suicide, self-harm, and psychological disorder among young offenders in custody. Journal of Correctional Health Care, 21(3), 243-254. https://doi.org/10.1177/ 1078345815584849

National Action Alliance for Suicide Prevention: Youth in Contact with the Juvenile Justice System Task Force. (2013). Suicidal ideation and behavior among youth in the juvenile justice system: A review of the literature. Washington, DC.

Paillé, P. (1994). L'analyse par théorisation ancrée. Cabiers de Recherche Sociologique, (23), 147-181. https://doi.org/10.7202/1002253ar

Paquette, D., Laporte, L., Bigras, M. et Zoccolillo, M. (2004). Validation de la version française du CTQ et prévalence de l'histoire de maltraitance 1. Santé mentale au Québec, 29(1), 201-220.

Petering, R. (2016). Sexual risk, substance use, mental health, and trauma experiences of gang-involved homeless youth. Journal of Adolescence, 48, 73-81. https://doi.org/10.1016/j.adolescence.2016.01.009

Piquero, A. R., Farrington, D. P. et Blumstein, A. (2003). The criminal career paradigm. Crime and Justice, 30, 359-506. https://doi.org/10.1086/652234

Radeloff, D., Lempp, T., Herrmann, E., Kettner, M., Bennefeld-Kersten, K. et Freitag, C. M. (2015). National total survey of German adolescent suicide in prison. European Child $\mathfrak{W}$ Adolescent Psychiatry, 24(2), 219-225. https:// doi.org/10.1007/s00787-014-0568-1

Reitano, J. (2017). Statistiques sur les services correctionnels pour adultes au Canada, 2015-2016 (Rapport n 85-002-X). Repéré à http://www.statcan.gc.ca/ $\mathrm{pub} / 85-002-\mathrm{x} / 2017001 /$ article/14700-fra.pdf

Rolland, J. P., Parker, W. D. et Stumpf, H. (1998). A psychometric examination of the French translations of NEO-PI-R and NEO-FFI. Journal of Personality Assessment, 71(2), 269-291. https://doi.org/10.1207/s15327752jpa7102_13

Schwalbe, C. S., Gearing, R. E., Mackenzie, M. J., Brewer, K. B. et Ibrahim, R. W. (2013). The impact of length of placement on self-reported mental health problems in detained Jordanian youth. International Journal of Law $\mho \sim P$ Psychiatry, 36(2), 107-112. https://doi.org/10.1016/j.ijlp.2013.01.003

Sedlak, A. J. et McPherson, K. S. (2010). Survey of youth in residential placement: Youth's needs and services. SYRP Report. Rockville, MD: Westat.

Shelton, D. (2004). Experiences of detained young offenders in need of mental health care. Journal of Nursing Scholarship, 36(2), 129-133. https://doi. org/10.1111/j.1547-5069.2004.04025.x

Soni, A. (2010). The relationship between delinquency and suicidality among adolescents (Thèse de doctorat inédite). Adler School of Professional Psychology, Chicago, IL.

Speckens, A. E. et Hawton, K. (2005). Social problem solving in adolescents with suicidal behavior: A systematic review. Suicide and Life-Threatening Behavior, 35(4), 365-387.

Stokes, M. L., McCoy, K. P., Abram, K. M., Byck, G. R. et Teplin, L. A. (2015). Suicidal ideation and behavior in youth in the juvenile justice system: a review of the literature. Journal of Correctional Health Care, 21(3), 222-242. 
St-Pierre, L. et Laurier, C. (2018). Vulnérabilité suicidaire des contrevenants en centre de réadaptation: présentation d'un modèle explicatif. Criminologie, 51(2), p. 264-287.

Strauss, A. L. et Corbin, J. (1990). Basics of qualitative research: Grounded theory procedures and techniques. Thousand Oaks, CA: Sage Publications.

Suk, E., Van Mill, J., Vermeiren, R., Ruchkin, V., Schwab-Stone, M., Doreleijers, T. et Deboutte, D. (2009). Adolescent suicidal ideation: a comparison of incarcerated and school-based samples. European Child $\mathfrak{G}$ Adolescent Psychiatry, 18(6), 377-383.

Taylor, T. J., Peterson, D., Esbensen, F.-A. et Freng, A. (2007). Gang membership as a risk factor for adolescent violent victimization. Journal of Research in Crime and Delinquency, 44(4), 351-380. https://doi.org/10.1177/ 0022427807305845

Thibodeau, L. et Perron, P.-A. (2017). La mortalité par suicide au Québec: 1981 à 2014 -Mise à jour 2017 (Rapport de surveillance). Institut national de santé publique du Québec. Repéré à http://collections.banq.qc.ca/ark: /52327/2773038

Viljoen, J. L., O’Neill, M. L. et Sidhu, A. (2005). Bullying behaviors in female and male adolescent offenders: Prevalence, types, and association with psychosocial adjustment. Aggressive Behavior, 31(6), 521-536. https://doi. org/10.1002/ab.20036

Wasserman, G. A., McReynolds, L. S., Schwalbe, C. S., Keating, J. M. et Jones, S. A. (2010). Psychiatric disorder, comorbidity, and suicidal behavior in juvenile justice youth. Criminal Justice and Behavior, 37(12), 1361-1376.

Williams, V., Grisso, T., Valentine, M. et Remsburg, N. (2008). Mental health screening: Pennsylvania's experience in juvenile detention. Repéré à http:// escholarship.umassmed.edu/psych_cmhsr/380/

\section{Young offenders at a crossroads: A study of their suicide risk}

ABSTRACT - The high level of antisocial behavior among young offenders in general and those associated with street gangs in particular, which accounts for their involvement with the justice system, has led to a lack of attention to their victimization and its psychological aftereffects. In this article, 212 young offenders in the care of youth services or correctional services were assessed using quantitative questionnaires and a semi-structured qualitative interview. The questionnaire was used to identify the variables most involved in suicide risk, assessed by two tools (MAYSI-2 and MINI). The qualitative interviews highlighted that young offenders in care find themselves at a crossroads where their desire to leave the delinquent life style confronts the feeling that their extremely restricted future possibilities will make this impossible. The discussion combines the results of the quantitative and qualitative parts, using an abductive approach to propose an integrated understanding of this phenomena. Finally, some suggestions for intervention are offered.

KEYWORDS - Young offenders, risk of suicide, mixed methods, mental health, assessment. 


\section{Jóvenes delincuentes en la encrucijada. Estudio de estimación mixta del riesgo suicida}

RESUMEN - Por causa de la predominancia de las conductas antisociales de los jóvenes delincuentes, en general, y de los asociados a las pandillas, particularmente, que constituyen, de hecho, el motivo de su acogida por el sistema judicial, es menos frecuente interesarse por su victimización y por sus secuelas psicológicas. Así, en el marco de este artículo, 212 jóvenes delincuentes a cargo de los servicios para jóvenes o de los servicios correccionales, fueron evaluados por medio de cuestionarios cuantitativos, y durante una entrevista cualitativa semi estructurada. Este estudio con metodología mixta permitió identificar, en la parte cuantitativa, las variables que más contribuyen estadísticamente al riesgo suicida, tal y como fue evaluado gracias a dos instrumentos (MAYSI-2 y MINI). Además, las entrevistas cualitativas evidenciaron que los jóvenes delincuentes a cargo, se encuentran en una intersección entre los caminos dónde se confronta el deseo de dejar un modo de funcionamiento delincuente, y el sentimiento de no poder hacerlo, por causa de un futuro considerado como si estuviera bloqueado. En la parte discusión, los resultados de los métodos cuantitativos y cualitativos son relacionados, con un procedimiento abductivo, para proponer una comprensión integrada. Finalmente, algunas sugerencias para la intervención completan este artículo.

PALABRAS CLAVE - Jóvenes delincuentes, riesgo suicida, método mixto, salud mental, evaluación. 